\title{
Fractura segmentaria abierta Illa de húmero en paciente lactante. Reporte de un caso
}

\section{IIla Open Segmental Fracture of the Humerus in an Infant Patient. A Case Report}

\author{
Milad Al Troudy El Troudi ${ }^{1}$ Marggiori K. Molina Garcia ${ }^{1}$ Manuel Brito ${ }^{2}$ \\ ${ }^{1}$ Medico Cirujano, Facultad de Medicina, Universidad de Los Andes, \\ Mérida, Estado Mérida, Venezuela \\ ${ }^{2}$ Cirujano Ortopédico y Traumatólogo, Laboratorio de Investigación \\ en Cirugía Ortopédica y Traumatología de la Universidad de Los \\ Andes, Mérida, Estado Mérida, Venezuela \\ Rev Chil Ortop Traumatol 2017;58:28-32. \\ Address for correspondence Dr. Milad Al Troudy El Troudi, MD, 2700 \\ SW 27TH AVE. APT 402 Miami, FL 33133 \\ (e-mail: miladaltroudy1@gmail.com).
}

\section{Resumen \\ Palabras Clave \\ - alta energía \\ - fracturas abiertas \\ - fracturas en niños}

Las fracturas diafisiarias de húmero constituyen solo el $3 \%$ de todas las fracturas en niños. Las fracturas abiertas de este segmento son poco frecuentes con muy pocos reportes en la literatura. Son el resultado de traumatismos de alta energía ocasionados por accidentes automovilísticos o precipitaciones de gran altura. Las fracturas abiertas en niños menores de 3 años, representa un verdadero reto y requieren un enfoque único y multidisciplinario. Las fracturas abiertas en los niños presentan desafíos especiales, donde deben considerarse las características especiales del hueso del niño que lo diferencian del hueso del adulto. La aplicación de los principios adecuados en el manejo de las fracturas abiertas, así como el establecimiento de protocolos específicos de tratamiento, son necesarios para garantizar una evolución adecuada de esas lesiones. Presentamos el caso de paciente lactante menor masculino, quien fue eyectado del asiento delantero de un vehículo rustico al volcarse éste por una pendiente, posterior a lo cual presenta fractura segmentaria abierta Illa del húmero derecho. El paciente es llevado a quirófano de emergencia donde se realiza limpieza quirúrgica y la estabilización de la fractura con un mini fijador externo utilizado para el tratamiento de fracturas de muñeca en adultos. Presentamos su tratamiento, complicaciones y evolución a corto plazo.

The humeral shaft fractures accounts for only 3\% of all fractures in children. Open fractures of this segment are rare with few reports in the literature. They result from high energy trauma caused by car accidents or precipitation from high altitude. Open fractures in children under 3 years represents a real challenge and requires a unique and multidisciplinary approach. We present male infant patient, who was ejected from the front seat of a rustic vehicle that overturned down a slope and presented an open segmental type Illa fracture of the right humerus. The patient was taken to the operating room where an emergency surgical cleaning and stabilization of the fracture was performed using a mini external fixator currently utilized for treating adult wrist fractures. We present the treatment, complications and outcomes in the short term. received

September 30, 2016

accepted

December 2, 2016
DOI http://dx.doi.org/ $10.1055 / \mathrm{s}-0037-1599080$. ISSN 0716-4548.
Copyright (e) 2017 by Thieme-Revinter

Publicações Ltda, Rio de Janeiro, Brazil
License terms

(c) $(1) \$$ 


\section{Introducción}

El sistema músculo-esquelético del paciente pediátrico presenta grandes diferencias con respecto al del adolescente y el adulto en muchas formas. La composición física y química del hueso en el infante, el periostio más activo y grueso y la presencia de placas de crecimiento, proporcionan mayor estabilidad y conducen consolidaciones más rápidas y confiables, que incluso permite la reconstitución de hueso en casos de defectos óseos importantes. ${ }^{1-5}$

Las fracturas abiertas en los niños, son el resultado de traumatismos de muy alta energía, y en muchos casos son el resultado de accidentes por vehículos automotores. ${ }^{2,6}$

Las complicaciones más frecuentes son las lesiones vasculares y nerviosas, discrepancias de longitud, consolidación viciosa, síndrome compartimental e infecciones. Sin embargo, las tasas de infección reportadas en niños con fracturas abiertas son más bajas que aquellas reportadas en adultos con tales fracturas. ${ }^{3,7,8}$

Las fracturas diafisiarias del húmero representan menos del 3\% de todas las fracturas en la edad pediátrica. ${ }^{6,7}$ Los reportes de fracturas abiertas en este segmento son escasos, con muy pocos casos publicados sobre fracturas abiertas del húmero en niños con edades inferiores a los 3 años. ${ }^{2-4,7}$

Presentamos el caso de paciente lactante masculino de 14 meses de edad, quien posteriormente al accidente automovilístico, presenta traumatismo en el brazo derecho complicado con fractura segmentaria abierta IIla de humero.

\section{Caso Clínico}

Paciente Masculino de 14 meses de edad, natural y procedente de los pueblos del sur, Estado Mérida, quien posteriormente al accidente automovilístico por el volcamiento de vehículo rustico en el que se desplazaba como pasajero en el asiento delantero con su madre, por una pendiente de una zona rural, es eyectado a través del parabrisas, sufriendo múltiples traumatismos, motivo por el cual es trasladado en ambulancia a la emergencia pediátrica de nuestro hospital.

El paciente fue evaluado de acuerdo a los protocolos del Pediatric Advanced Life Support (PALS) ${ }^{9}$ y del Advanced Trauma Life Support (ATLS). ${ }^{10} \mathrm{Al}$ examen físico de ingreso se registra tensión arterial 96/72 $\mathrm{mm} \mathrm{Hg}$, frecuencia cardíaca102 lpm y frecuencia respiratoria $28 \mathrm{rpm}$. Se encontraba pálido, sudoroso, afebril al tacto e hidratado. Normo céfalo, cuello móvil, simétrico, sin adenopatías. Tórax simétrico, normo expansible, sin alteraciones. Abdomen, blando, depresible, doloroso a la palpación. Miembro superior derecho con herida extensa en el hombro y cara antero externa del brazo, en relación con deformidad e impotencia funcional (-Fig. 1A). Pulsos distales presentes. Glasgow 15/ 15 puntos. Los estudios de laboratorio reportaron hemoglobina de $8,5 \mathrm{mg} / \mathrm{dL}$.

Se tomó un acceso venoso periférico y se inició la resucitación con fluidos, se administró Cefazolina $(100 \mathrm{mg} / \mathrm{kg})$, Gentamicina $(5 \mathrm{mg} / \mathrm{kg})$ y penicilina cristalina (150.000 UI/ $\mathrm{Kg}$ ) por vía endovenosa.

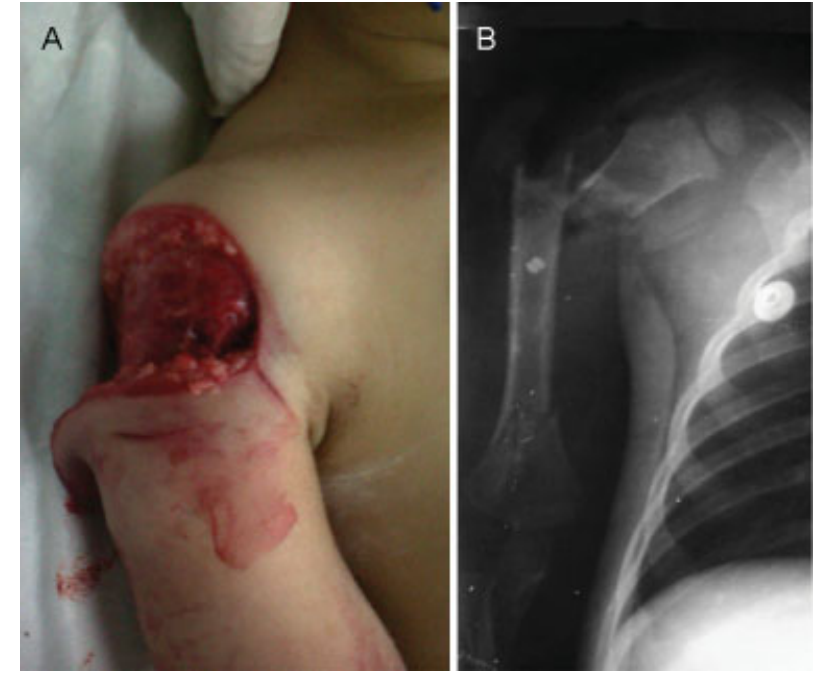

Fig. 1 Foto clínica al momento de su ingreso (A). Radiografía anteroposterior del brazo derecho a su ingreso (B).

Una vez estabilizado es llevado a la sala de estudios imagenológicos donde se realiza ultrasonografía que no reporta alteraciones para el momento de su realización; y radiografía simple anteroposterior del hombro y miembro superior derecho, donde se aprecia solución de continuidad ósea proximal y metafisiaria distal en el húmero derecho con desplazamiento en valgo (-Fig. 1B).

Fue valorado por el servicio de cirugía ortopédica y traumatología, quienes colocaron apósitos estériles en la herida, e inmovilizaron temporalmente con una férula de yeso, previa tracción y alineación de la extremidad para evitar tensión de las partes blandas.

Se ingresó con los diagnósticos de: 1) Politraumatizado: 1.1 Traumatismo encéfalo craneal leve; 1.2 Traumatismo abdominal no complicado; 1.3 Fractura segmentaria abierta IIIa Gustilo y Anderson ${ }^{11}$ de humero derecho; 1.4 Síndrome Anémico Agudo.

Es llevado a quirófano de emergencia el mismo día de su ingreso donde se realiza irrigación con aproximadamente 7.000 cc de solución $0,9 \%$, desbridamiento extenso de piel y músculos desvitalizados, reducción indirecta, osteodesis con alambres de Kirschner y fijación externa con un fijador externo modular para fracturas de muñeca del adulto. (-Fig. 2).

La antibioticoterapia se continuó con las dosis antes mencionadas de cefazolina y gentamicina cada 8 horas. La penicilina sólo se utilizó al momento de su ingreso para prevenir infecciones por Clostridium y anaerobios por el riesgo de la zona en que ocurrió el accidente.

Posteriormente es llevado de forma sucesiva a quirófano para realizar exploración quirúrgica y lavado de la herida, en cuatro ocasiones durante un mes. Una vez delimitada un área de piel necrótica es llevado a quirófano y se realiza la necrectomía de la misma. A cuatro semanas de su ingreso y una comprobada la ausencia de procesos infecciosos se realiza un injerto dermo-epidérmico por el servicio de cirugía ortopédica y traumatología (-Fig. 3). 


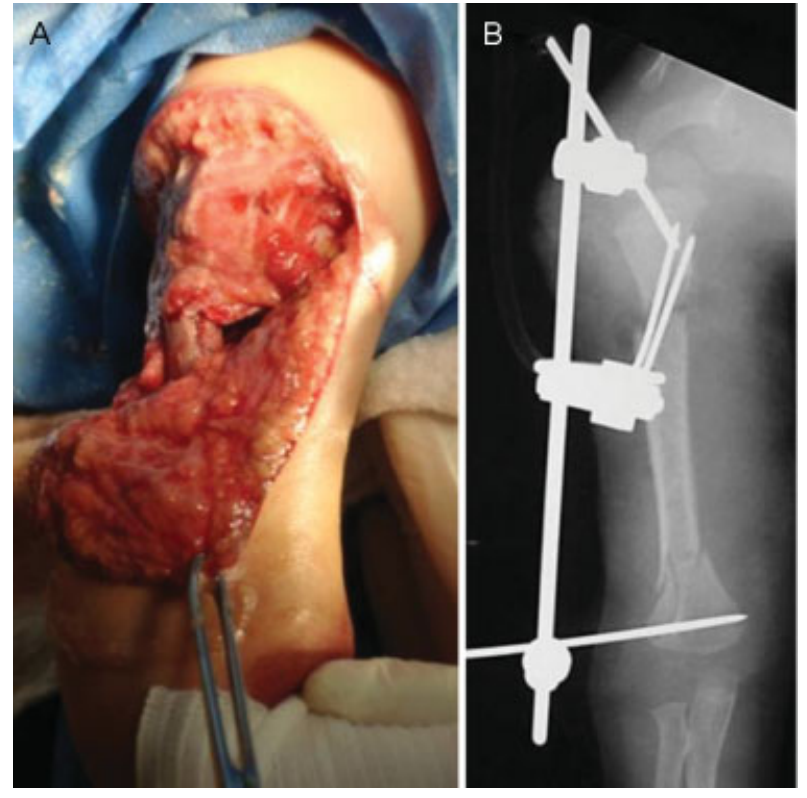

Fig. 2 Foto clínica intraoperatoria de la exploración quirúrgica al ingreso (A). Radiografía anteroposterior del brazo derecho posterior a su estabilización con fijación externa (B).

El fijador externo es retirado a las 8 semanas del ingreso, una vez evidenciada la consolidación de la fractura y la integración completa del injerto ( - Figs. 4 y 5). No se evidenciaron alteraciones funcionales o limitaciones importantes en la movilización del hombro y el codo en el seguimiento a corto plazo, correspondiente a los 6 meses subsiguientes a la cirugía, en los que se realizaron los estudios radiológicos y funcionales de control.

\section{Discusión}

Las lesiones causadas por accidentes de tránsito constituyen un importante problema de salud pública, cuya prevención eficaz y sostenible exige esfuerzos concertados. Se estima que, cada año, en el mundo mueren 1,2 millones de personas a causa de choques en la vía pública y hasta 50 millones resultan heridos, con un importante impacto social y económico sobre todo en países en vías de desarrollo. ${ }^{12,13}$
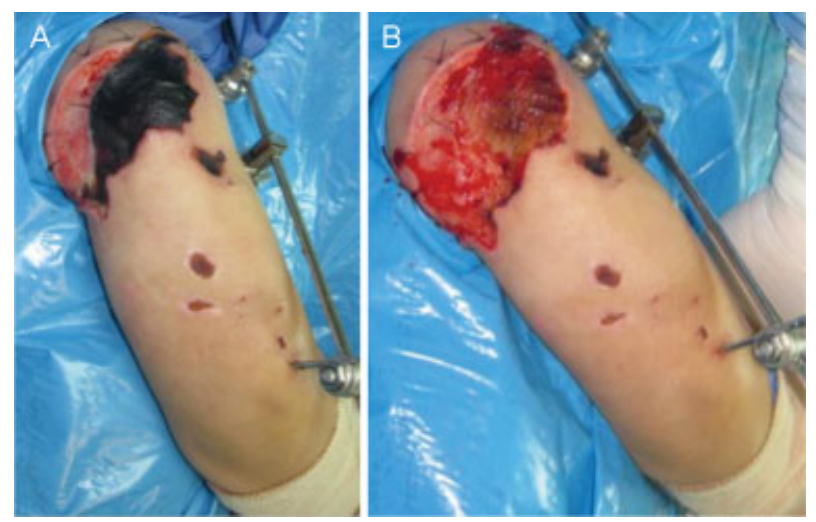

Fig. 3 Foto clínica donde se aprecia el área necrótica de la herida (A). Foto clínica intraoperatoria tras ser realizada la necrectomía (B).

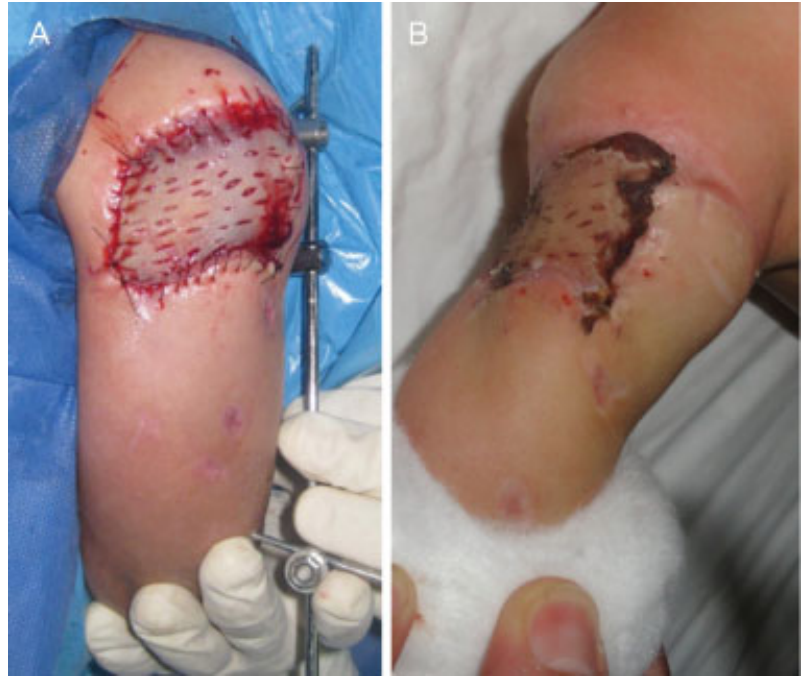

Fig. 4 Foto clínica posterior a la realización del injerto dermoepidérmico (A). Foto clínica del injerto tras su integración completa a las 8 semanas (B).

Las fracturas abiertas por accidentes automovilísticos en niños, sobre todo en aquellos con edades inferiores a los 3 años, son muy poco frecuentes. Sin embargo, las proyecciones indican que, sin un renovado compromiso con la prevención, estas cifras aumentarán en torno al 65\% en los próximos 20 años. $^{13}$

En los niños, las fracturas del antebrazo y la tibia son las más frecuentes. Skaggs et $\mathrm{al}^{14}$ en un estudio multicéntrico de 554 fracturas abiertas en niños, encontró que los sitios más frecuentes fueron la tibia o el peroné (34\%), radio o cúbito



Fig. 5 Radiografías anteroposterior y lateral del húmero derecho a 8 semanas del ingreso, donde se evidencia la consolidación completa de ambas fracturas. 
(32\%), mano o metacarpianos (10\%), fémur (6,7\%) y húmero $(6,5 \%)$.

La clasificación para las fracturas abiertas más conocida y utilizada es la de Gustilo y Anderson, ${ }^{11}$ tanto para adultos como para niños. Toma en cuenta el mecanismo de producción, la gravedad del daño de los tejidos blandos, el patrón de fractura y su riesgo de contaminación. Tiene la ventaja de servir como guía para el tratamiento y evaluación de los resultados a mediano y largo plazo.

Según esta clasificación, las fracturas Tipo III, son fracturas asociadas a heridas superiores a los $10 \mathrm{~cm}$, con muy alto riesgo de contaminación, producidas por traumatismos de muy alta energía. Las subtipo IIIa, que nos concierne en este caso, son aquellas que tienen una adecuada cobertura de los tejidos blandos tras el desbridamiento inicial.

Haasbeek y Cole ${ }^{15}$ en una revisión de 61 fracturas abiertas del miembro superior, reportó 15 (24,59\%) casos de fracturas abiertas del húmero, de las cuales $2(3,27 \%)$ se correspondían con el tipo III.

Los factores que determinan el tratamiento y su éxito en los niños son la edad; peso; tipo de fractura; hueso y segmento afectado; manejo operatorio o no operatorio; la disponibilidad de los implantes adecuados; la experiencia del cirujano; el manejo postoperatorio y la relación costo/beneficio. ${ }^{2}$

En nuestro caso, la evaluación y el manejo inicial, la elección de la antibioticoterapia, la irrigación y desbridamiento temprano y la estabilización de la fractura, permitieron la buena evolución de las heridas y la curación temprana de las partes blandas y el hueso. La fijación externa es un método ampliamente recomendado para el tratamiento de estas fracturas y el "control de daños" es un concepto muy bien reconocido en el manejo ortopédico del trauma del adulto. Con el aumento de la frecuencia de las fracturas abiertas en niños, la aplicación de estas técnicas, como en el caso que presentamos, puede mejorar los resultados y evitar las complicaciones tardías. ${ }^{8}$

Nuestro caso tuvo un seguimiento a corto plazo de 6 meses posterior a la cirugía, con una periodicidad mensual, motivado por la negativa del representante del infante de continuar asistiendo a los controles. Sin embargo, el primer trimestre se caracterizó por una evolución favorable aunque lenta debido a la edad del paciente lo cual condicionaba un factor adverso a la recuperación de los tejidos blandos y óseos. Se realizaron estudios radiográficos control en cada chequeo con evolución favorable respecto a la consolidación ósea. Para el final del primer trimestre postoperatorio el rango de movimiento del miembro afecto se encontraba alrededor del 50\%. Para el sexto mes, el control radiológico reportó la consolidación total aunque con la formación de callo óseo característico. De igual manera, el rango de movimiento al final del semestre posterior al evento se encontraba alrededor del 90\%, notándose la limitación residual del brazo en su rotación. Sin embargo, el rango de movimiento del hombro y codo se restituyeron en su totalidad para el final del periodo de meses.

A pesar de las limitantes, este caso ilustró cómo la aplicación del "control de daños", un puente temporal en el manejo de una fractura de húmero abierta, segmentaria, con un importante compromiso de las partes blandas y del hueso, permitió su buena evolución a corto plazo (6 meses de seguimiento y control), minimizando las complicaciones. Sin embargo, es necesario que se fabriquen implantes diseñados para el manejo de ese tipo de lesiones, sobre todo en aquellos más pequeños.

\section{Referencias}

1 Carson S, Woolridge DP, Colletti J, Kilgore K. Pediatric upper extremity injuries. Pediatr Clin North Am 2006;53(01):41-67, v

2 Slongo TF. Fracture treatment in childhood. Injury 2005;36 (Suppl 1):A1

3 Stewart DG Jr, Kay RM, Skaggs DL. Open fractures in children. Principles of evaluation and management. J Bone Joint Surg Am 2005;87(12):2784-2798

4 Rodríguez-Merchán EC. Pediatric skeletal trauma: a review and historical perspective. Clin Orthop Relat Res 2005;(432):8-13

5 Canale S. Campbell: Cirugía Ortopédica. 11 ed. Madrid: Elsevier; 2011:3237-62

6 Beaty J, Kasser J. Rockwood y Wilkins's. Fracturas en el niño. 5ta Ed Madrid: Marbán; 2007

7 Caviglia H, Garrido CP, Palazzi FF, Meana NV. Pediatric fractures of the humerus. Clin Orthop Relat Res 2005;(432):49-56

8 Mooney JF. The use of 'damage control orthopedics' techniques in children with segmental open femur fractures. J Pediatr Orthop B 2012;21(05):400-403

9 Pandya NK, Upasani VV, Kulkarni VA. The pediatric polytrauma patient: current concepts. J Am Acad Orthop Surg 2013;21(03): 170-179

10 American College of Surgeons, Committee on Trauma. Advanced trauma life support manual. Chicago: American College of Surgeons. 1997

11 Gustilo RB, Mendoza RM, Williams DN. Problems in the management of type III (severe) open fractures: a new classification of type III open fractures. J Trauma 1984;24(08):742-746

12 Organización Mundial de la Salud / Departamento de Prevención de la Violencia y los Traumatismos y Discapacidad. Plan Mundial para el Decenio de Acción para la Seguridad Vial 2011-2020. Génova: Organización Mundial de la Salud; 2011

13 Peden M. et al. The world report on road traffic injury prevention. Ginebra: Organización Mundial de la Salud; 2004

14 Skaggs DL, Friend L, Alman B, et al. The effect of surgical delay on acute infection following 554 open fractures in children. J Bone Joint Surg Am 2005;87(01):8-12

15 Haasbeek JF, Cole WG. Open fractures of the arm in children. J Bone Joint Surg Br 1995;77(04):576-581 